\title{
ALBERT JAMES ARNOLD, La littérature antillaise entre histoire et mémoire. 1935-1995
}

\section{Carminella Biondi}

\section{(2) OpenEdition}

\section{Journals}

\section{Édition électronique}

URL : https://journals.openedition.org/studifrancesi/44438

DOI : $10.4000 /$ studifrancesi.44438

ISSN : 2427-5856

\section{Éditeur}

Rosenberg \& Sellier

\section{Édition imprimée}

Date de publication : 1 juin 2021

Pagination : 256-257

ISSN : 0039-2944

\section{Référence électronique}

Carminella Biondi, « ALBERT JAMES ARNOLD, La littérature antillaise entre histoire et mémoire. 1935-1995 »,

Studi Francesi [En ligne], 193 (LXV | I) | 2021, mis en ligne le 01 juillet 2021, consulté le 15 octobre

2022. URL : http://journals.openedition.org/studifrancesi/44438 ; DOI : https://doi.org/10.4000/ studifrancesi.44438

Ce document a été généré automatiquement le 15 octobre 2022

\section{cc) (i) (9)}

Creative Commons - Attribution - Pas d'Utilisation Commerciale - Pas de Modification 4.0 International - CC BY-NC-ND 4.0

https://creativecommons.org/licenses/by-nc-nd/4.0/ 


\title{
ALBERT JAMES ARNOLD, La littérature antillaise entre histoire et mémoire. 1935-1995
}

\author{
Carminella Biondi
}

\section{RÉFÉRENCE}

ALBERT JAMES ARNOLD, La littérature antillaise entre histoire et mémoire. 1935-1995, Paris, Classiques Garnier, «Bibliothèques francophones» 9, 2020, 354 pp.

1 Le titre indique de façon très nette que celle qui nous est proposée ici ne veut pas être une histoire de la littérature antillaise, et les dates posent des limites temporelles bien précises à l'objet dont il est question. Mais si le contenu correspond à ce que nous suggère le titre choisi, car le fil rouge qui unit les textes examinés est en effet un dialogue serré entre histoire et mémoire et les dates indiquées limitent la période qui est au cœur de la recherche, il faut reconnaître qu'il s'agit bel et bien d'une histoire de la littérature antillaise, dans le sens le plus haut et le plus positif du terme, car grâce à des liens souterrains et à des renvois savants, l'auteur nous fait suivre le lent cheminement de cette littérature depuis les origines (dans un Prologue qui pose les bases des problématiques futures, l'auteur s'arrête, à juste titre, sur le Code Noir) jusqu'à nos jours. Le choix des auteurs et des textes ne suit pas une chronologie rigide et n'a aucune prétention à l'exhaustivité, mais un lien se tisse en profondeur qui relie entre eux tous les ouvrages étudiés, le long d'un parcours dont l'auteur fixe très clairement les étapes, bien enracinées dans leur contexte historique. Un contexte qui n'est pas seulement celui des Antilles françaises, mais qui s'élargit, même si de manière rapide, à toute l'aire de la Caraibe: «Nos échanges avec d'éminents spécialistes des littératures anglophone, francophone, hispanophone et néerlandophone ont ouvert des perspectives nouvelles et ont permis de contextualiser la place des Antilles Françaises dans l'aire culturelle qu'elles partagent avec ces littératures caribéennes. La formation 
de l'imaginaire antillais nous semble, dans cette perspective comparatiste, exceptionnelle» (Introduction, p. 7).

Personne mieux que l'auteur de cette Littérature ne pouvait mener à bien une entreprise qui, pour éviter les pièges où tombent parfois les comparatistes, doit se fonder sur des connaissances sédimentées dans le temps: les recherches d'Albert James Arnold datent des années 1980 et, entre la fin du xxe et le début du xxie siècle, il a coordonné une History of Literature in the Caribbean (1994-2001) en trois volumes, dont le troisième porte un titre éloquent: Cross-Cultural Studies.

3 Le volume est divisé en trois parties qui indiquent les étapes d'un parcours dont les aboutissements sont incertains: I- «Formation de l'imaginaire antillais»; II- «Éléments du dilemme»; III- «Sortir de l'impasse?» La première partie s'ouvre sur un long Prologue, à la recherche, dans un passé lointain, des éléments qui peuvent être encore utilisables au $\mathrm{xx}^{\mathrm{e}}$ siècle, ainsi que l'auteur l'indique de façon explicite dans le titre du paragraphe consacré à l'Histoire des Aventuriers, des Boucaniers et de la Chambre des comptes établie dans les Indes d'Oexmelin ou Exmelin (1686 dans sa version française): «Exmelin et la recherche d'un passé utilisable au xx siècle», ou bien dans celui où il est question de la Pariade (accouplement des marins avec les femmes esclaves sur le bateau négrier): «Une scène fondatrice du discours identitaire: la Pariade». La première partie se clôt sur un dilemme qui ne cesse de hanter et de conditionner de manière plus ou moins évidente toute la littérature antillaise, encore de nos jours (l'auteur précise qu'il utilise l'adjectif "antillais" pour les Antilles françaises et "caribéen" pour toute l'aire de la Caraïbe): «Le rattachement toujours plus ferme des Antilles Françaises à la métropole depuis le milieu du $\mathrm{xx}^{\mathrm{e}}$ siècle constitue un dilemme pour les auteurs guadeloupéens et martiniquais» (p. 73). On le retrouve, ce dilemme, dans l'Épilogue qui conclut l'ouvrage, dont le titre pose une question cruciale: «Quel avenir pour les Régions monodépartementales?». Tandis que les habitants des Caraïbes indépendantes sont en train de forger leurs identités, ceux des Antilles françaises, après des tentatives d'indépendance qui remontent aux années 1970, ont indiqué clairement, dans les derniers referendums, leur volonté de rester liés à la France pour des raisons économiques. Arnold doit conclure sa recherche par cette constatation amère: «Dans ces conditions, on voit difficilement comment un sentiment national pourra se développer. Le devoir de mémoire a de beaux jours devant lui, la division des ethnoclasses servant à l'entretenir et à préserver les conflits historiques sans espoir de résolution» (p. 318). Cette référence conclusive au «devoir de mémoire» nous rappelle l'enjeu qui est au cœur de l'ouvrage et qui marque les étapes du parcours de cette littérature, en particulier mais pas seulement au $\mathrm{xx}^{\mathrm{e}}$ siècle: le passage du devoir de mémoire, qui idéalise et fragmente le passé pour le besoin d'une cause ou d'une ethnoclasse, à celui «d'histoire», qui voudrait se fonder sur une reconstruction objective du passé, concernant tous les protagonistes qui y sont impliqués, une reconstruction qui ne veut pas séparer mais englober. Ces étapes, au $\mathrm{xx}^{\mathrm{e}}$ siècle, sont bien synthétisées dans le premier chapitre de la troisième partie: «Négritude, créolité, créolisation» qui, après avoir souligné l'apport incontournable de Glissant vers la dernière étape qui s'ouvre au Tout-monde, se termine par une analyse de Texaco (1992), où Patrick Chamoiseau «a entrepris d'utiliser les techniques de la mémoire au service de l'histoire», tout en évitant "d'entériner le découpage de l'Histoire (officielle)» (p. 243). Un parcours plein d'obstacles, qui se multiplient lorsque aux problèmes 
d'ethnoclasse s'ajoutent ceux de genre, étudiés dans le deuxième chapitre de la troisième partie: «Vers une créolisation au féminin en Guadeloupe» (pp. 253-311).

4 Mais tout ce dont j'ai parlé jusqu'ici n'est que la structure portante de l'ouvrage, à laquelle l'analyse détaillée des textes, très connus ou presque méconnus, donne une âme passionnée et passionnante. Je serais tentée d'affirmer, si cela ne pouvait paraître dévalorisant pour un ouvrage critique, que cet essai se lit comme un roman, comme la longue saga du peuple antillais dans son effort d'enracinement, et de ses écrivains qui se sont efforcés et s'efforcent de fonder sur des bases solides une "littérature antillaise". L'avenir littéraire des Antilles françaises, on l'a vu, reste toutefois incertain, car ces dernières sont encore acculées dans une impasse: «À la fois française, parce que relevant de l'une ou l'autre des Régions monodépartementales, et autre parce qu'elle est extra-hexagonale, la littérature antillaise répond nécessairement à deux pressions souvent contradictoires» (p. 316). Un long chemin reste à faire. 